\title{
A Novel Four Dimensional Hyper-chaotic Coupled Dynamos System and Its Tracking Control and Synchronization
}

\author{
Yanyun $\mathrm{Xie}^{1, *}$ and Wenliang $\mathrm{Cai}^{2}$ \\ Chongqing Water Resources and Electric Engineering College, Chongqing \\ Yongchuan 402160, China \\ ${ }^{1}$ xieyanyun2008@126.com, ${ }^{2}$ caiwenliang2008@126.com \\ * Corresponding Author
}

\begin{abstract}
This paper mainly investigates the tracking control and synchronization problems of the new four-dimensional hyper-chaotic coupled dynamos system. Design the suitable controller make the single variable or all variables of hyper chaos coupling generator system can track any reference signal; at the same time two system with the same structures and different structures can realize synchronizing. Lyapunov stability theory and numerical simulation showed the effectiveness of the two methods of the synchronization.
\end{abstract}

Keywords: Hyper-chaotic; Coupled dynamos system; Tracking control; Synchronization

\section{Introduction}

Chaos system is a kind of complex and irregular nonlinear dynamics behavior, its intrinsic randomness, sensitivity to initial value and continuous broad spectral characteristics are widely used in the image data encryption, secure communications and information technology. Since 1990 when OGY chaos control method was proposed by Ott [1] and when chaotic synchronization in electronic circuit was realized by Pecora and Carroll [2], chaos control and chaos synchronization have become one of the chaos research hot fields. Tracking control is a novel chaos control method in recent years, it enables any controlled system variable track any given reference signal. The controller of single variable tracking control method has a simple structure, it make tracking control is easy to implement. Because of with easy popularization, tracking control has been attracted a great deal of attention of many scholars. Chaotic synchronization is a generalized chaos control problem, and it is different with the traditional chaotic control method. In simple terms, chaotic synchronization is to control two chaotic systems keep with same step. It can make phase diagram coincidence of two chaotic systems by effective control method. As we have been known, Lixiang Li realizes the tracking of discrete chaotic system [3]; Xingyuan Wang realizes the tracking adaptive control and synchronization of chaotic coupled dynamos system [4]; Jiankui Peng realizes the analysis and tracking control of chaotic coupled dynamos system [5]; Yongjian Liu proposed the combined synchronization control of hyper chaotic system [6].

Hyper chaotic system has more complex dynamical behavior than the chaotic systems; hyper chaotic system make secure communication more safety, and therefore research on control and synchronization of hyper chaotic system has important theoretical and practical significance. Taking four-dimensional hyper chaotic coupling dynamos system as example, this paper studies random variables of hyper-chaotic system tracking any reference signals and implement the synchronize between two chaotic systems with the same structures and different structures, theoretical proof and numerical simulations show effectiveness of the two methods of the synchronization. 


\section{Four-Dimensional Hyper-Chaotic Coupled Dynamos System Model}

In order to generate hyper-chaotic coupled dynamos system. Form new fourdimensional hyper chaotic coupling generator system by increasing a non-linear controller $w$ in the three-dimensional coupling dynamos $\operatorname{system}^{[4,7-9]}$, the state equation is given as follows:

$$
\left\{\begin{array}{l}
\dot{x}=-2 x+y(z+3)+w \\
\dot{y}=-2 y+x(z-3) \\
\dot{z}=z-x y \\
\dot{w}=-m y
\end{array}\right.
$$

Where $\mathrm{x}, \mathrm{y}, \mathrm{z}, \mathrm{w}$ are state variables while $\mathrm{m}$ is parameter.

We can see from formula (1), the new system meets the necessary conditions to produce hyper-chaotic: three-dimensional coupling generator system became fourdimensional coupling generator system; and equation contains quadratic cross-product terms. The lyapunov exponents of the system (1) are depicted in Figure 1 when changing the value of $m$. Interestingly, when $m=2$ is selected, new system (1) is hyper-chaotic. In this case, the Lyapunov exponents of the $\operatorname{system}(1)$ are $\lambda_{1}=0.13>0, \lambda_{2}=0.06>0$

$\lambda_{3}=0$, and $\lambda_{4}=-3.13$ with the Wolf ${ }^{[10-11]}$ method. The projections of the hyperchaotic attractors about four-dimensional hyper-chaotic coupled dynamos system are illustrated in Figure 2 and the hyper-chaotic behavior of the system can be observed in Figure 3.

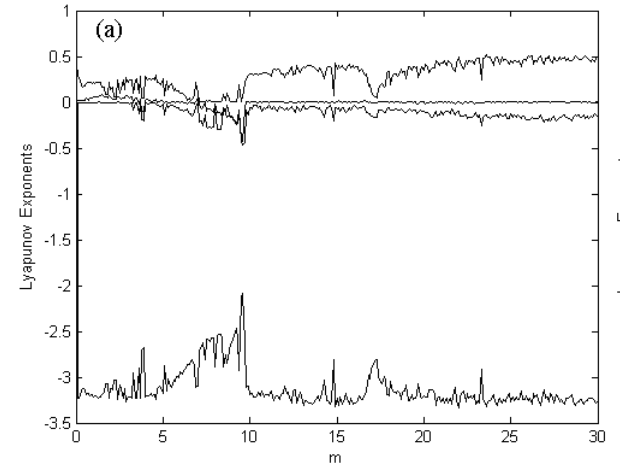

(a)



(b)

Figure 1. The Lyapunov Exponents of the System (1) with Respect to the Parameter $m$. (a) The Parameter $m$ in the Range [0, 30]; (b) The Parameter $m$ in the Range $[0.5,3]$ 


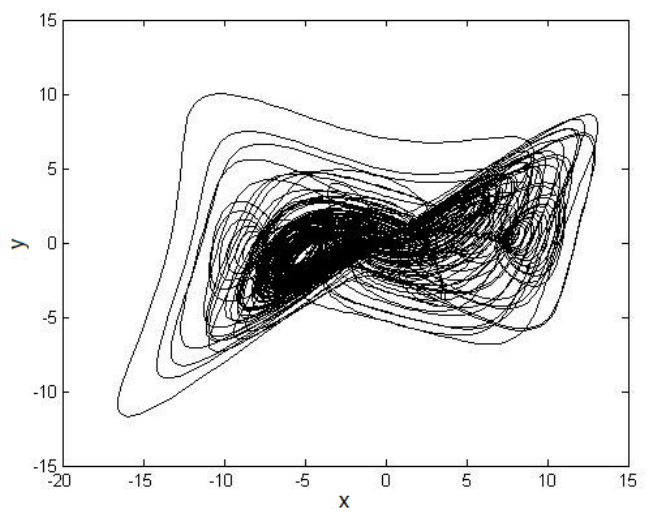

(a)

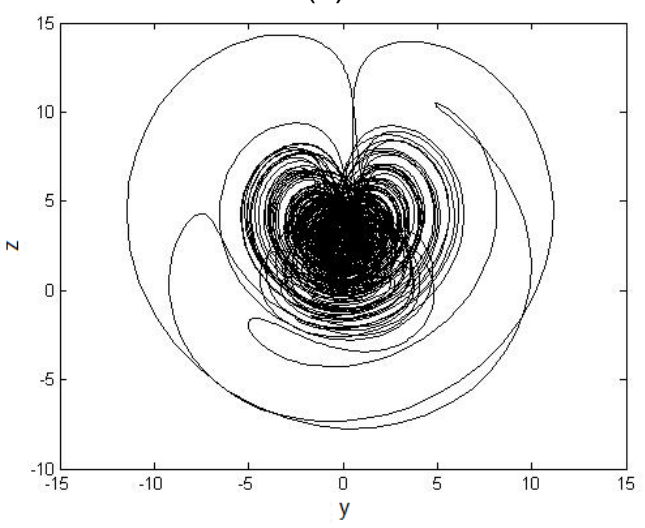

(c)

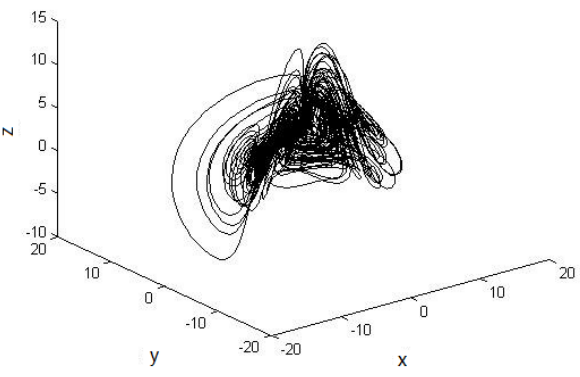

(e)

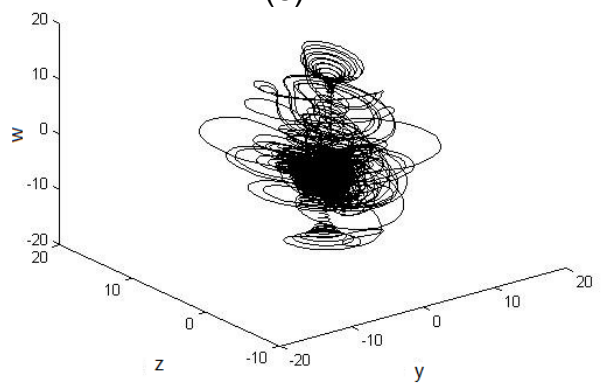

(g)

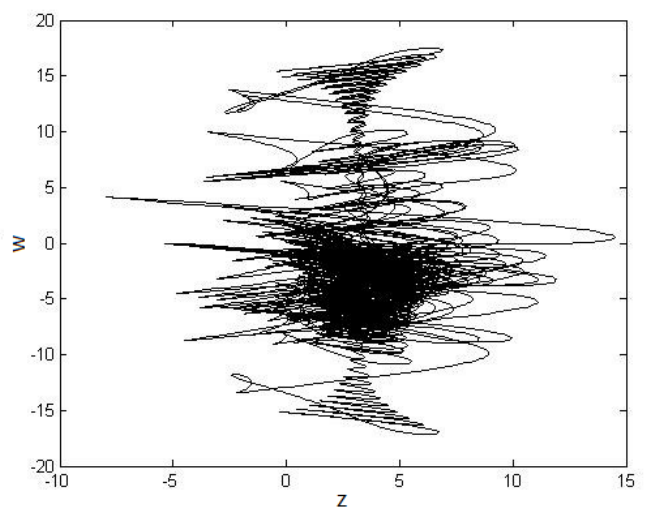

(b)

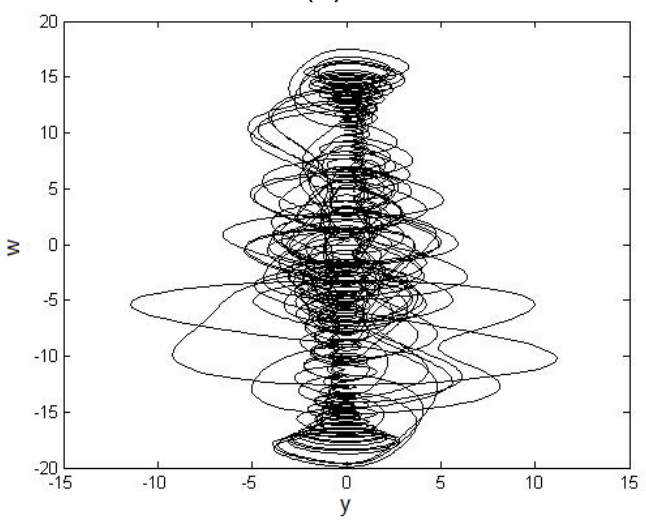

(d)

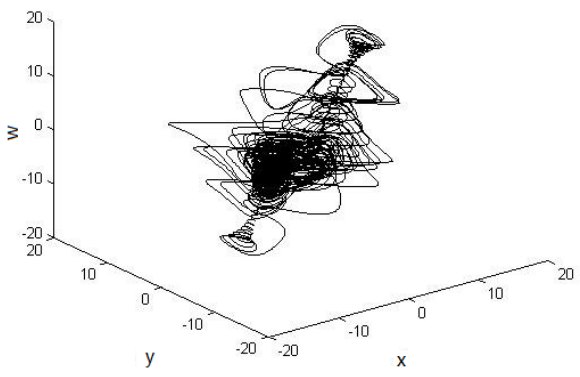

(f)

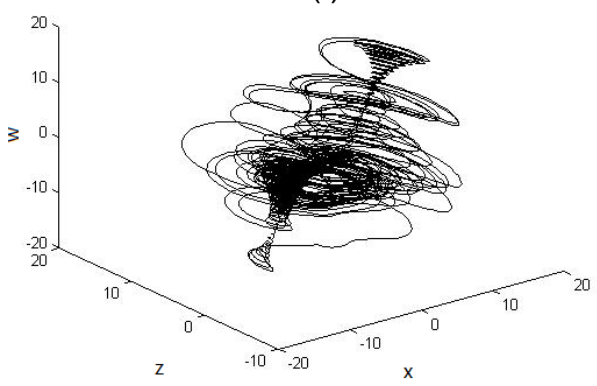

(h)

Figure 2. The Projection of the Hyper-chaotic Attractor of Four-dimensional Coupling Dynamos System (1) in (a) x-y Pane, (b) z-w Plane, (c) y-z Space, (d) y-w Space, (e) $x-y-z$ Space, (f) $x-y-w$ Space, (g) y-z-w Space, and (h) $x-z-w$ Space 


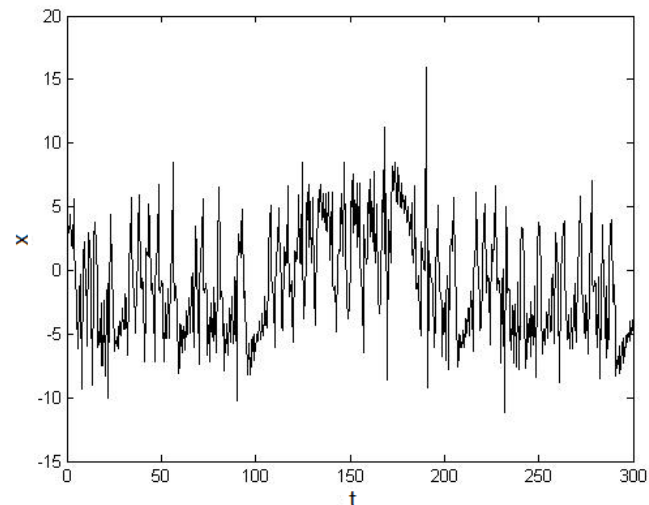

(a)

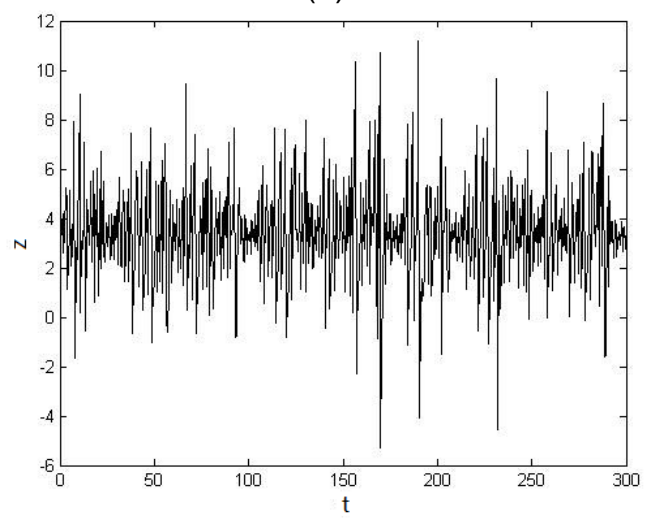

(c)

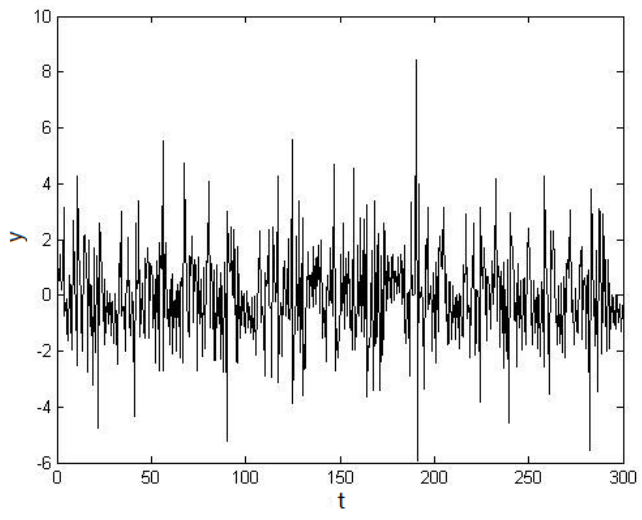

(b)

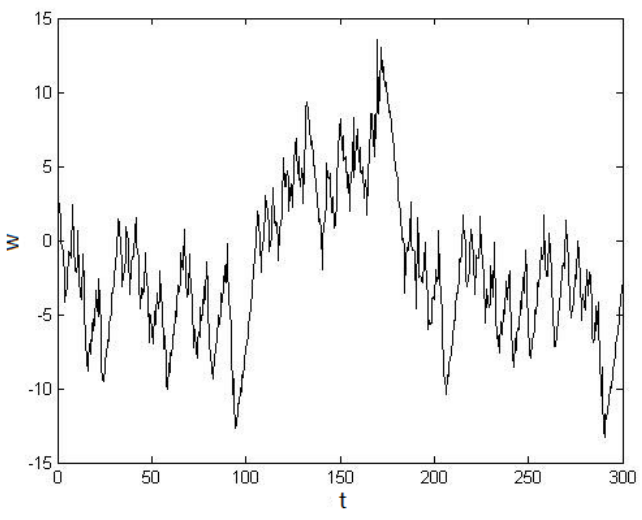

(d)

Figure 3. Time Response Diagram of System (1): (a) Sensitive Dependence on Initial Value of $\mathbf{x}$; (b) Sensitive Dependence on Initial Value of y; (c) Sensitive Dependence on Initial Value of z; (d) Sensitive Dependence on Initial Value of $\mathbf{w}$

At the same time, the concrete calculating methods of the lyapunov dimension of system (1) are as follows according to Kaplan-Yorke ${ }^{[12]}$ conjecture formula.

$$
D=j+\frac{1}{\left|\lambda_{j+1}\right|} \sum_{i=1}^{j} \lambda_{i}=3+\frac{0.13+0.06}{|-3.13|}=3.061
$$

This further illustrates the system is hyper-chaotic with above parameters.

\section{Tracking Control}

In this section, we investigate the single variable of hyper chaos coupling generator system tracks any reference signal by adaptive tracking control method, and the all variables of hyper-chaos coupling generator system track four signals by hybrid tracking control method.

\subsection{Adaptive Tracking Control}

To achieve adaptive tracking control of single variable in four-dimensional coupling generator system (1), design adaptive controller, the controlled system is given as below: 


$$
\left\{\begin{aligned}
\dot{x} & =-2 x+y(z+3)+w \\
\dot{y} & =-2 y+x(z-3)+u \\
\dot{z} & =z-x y \\
\dot{w} & =-2 y
\end{aligned}\right.
$$

Where $x, \mathrm{y}, z, w$ are the states and $u$ is the adaptive controller.

In order to selecting the state $w(t)$ of the four-dimensional coupling generator system (1) to tracking any reference signal $q(t)$, we define the tracking error state as

$$
e(t)=w(t)-q(t)
$$

If $e(t) \rightarrow 0$ as $\mathrm{t} \rightarrow \infty$, the state $w(t)$ and reference signal $q(t)$ are globally as asymptotically self-synchronization.

We select the adaptive controller which is defined by

$$
u=-\frac{k}{2}(e+\dot{e})^{2}+2 y-x(z-3)-\frac{\ddot{q}}{2}
$$

In which $k$ is the adaptive control gain and meet as $\dot{k}=-\mu(e+\dot{e})^{2}$ which $\mu$ is a positive constant.

One considers a Lyapunov function of the controlled system (2) as

$$
u(t)=\frac{1}{2} e^{2}+\frac{1}{2}(e+\dot{e})^{2}+\frac{1}{2 \mu}(k+1)^{2}
$$

The derivative of $u(t)$ along the solution of system (2) is

$$
\begin{gathered}
\frac{d u}{d t}=e \dot{e}+(e+\dot{e})(\dot{e}+\ddot{e})+\frac{1}{\mu}(k+1) \dot{k}=-e^{2}+(e+\dot{e})(e+\dot{e}+\ddot{w}-\ddot{q})-(k+1)(e+\dot{e})^{2} \\
=-e^{2}+(e+\dot{e})\{e+\dot{e}-2 \times[-2 y+x(z-3)+u]-\ddot{q}\}-(k+1)(e+\dot{e})^{2} \\
=-e^{2} \leq 0
\end{gathered}
$$

Since $\frac{d u}{d t}$ is a semi-negative definite function and $u(t)$ is a positive definite function, it is noting that the state $w(t)$ of the controlled system (2) can trace any reference signal $q(t)$ progressively according to Lyapunov stability theory.

If we choose any reference signal $q(t)$ as $q(t)=\sin t$, and system parameters $\mu=5$, the initial condition $(x(0), y(0), z(0), w(0))=(1,2,3,4)$, step-size $h=0.01$ are selected, the state ${ }^{w(t)}$ of the controlled system (2) can trace reference signal $q(t)$ progressively. The simulation results are illustrated in Figure 4 and Figure 5. It can be seen that the timehistory of tracking error will converge to zero in Figure 4. It shows that the state ${ }^{w(t)}$ of four-dimensional coupling generator system (2) tracking reference signal $\sin t$ is achieved after $\mathrm{t} \leq 5$ seconds in Figure 4. This means the validity and accuracy of the tracking using adaptive tracking control method. 


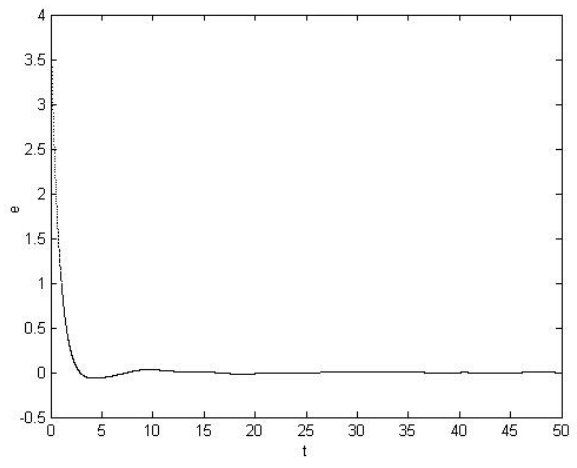

Figure 4. Time-History of Tracking Error Diagram

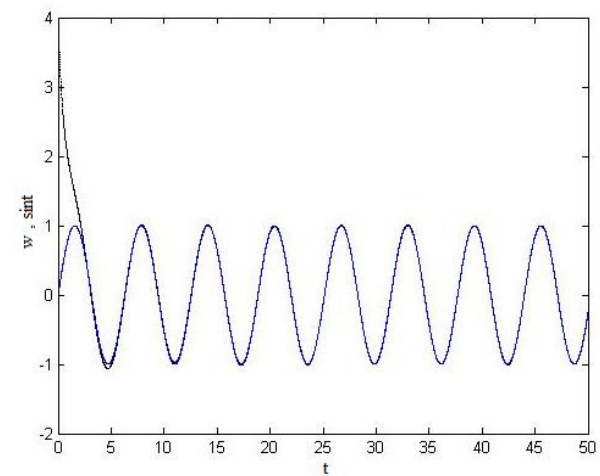

Figure 5. Time-Domain Waveform between $w$ and $\sin t$

\subsection{Hybrid Tracking Control}

To achieve hybrid tracking control of all variables in four-dimensional coupling generator system (1), a hybrid tracking controller is designed, the controlled system is given as below:

$$
\left\{\begin{array}{l}
\dot{x}=-2 x+y(z+3)+w+u_{1} \\
\dot{y}=-2 y+x(z-3)+u_{2} \\
\dot{z}=z-x y+u_{3} \\
\dot{w}=-m y+u_{4}
\end{array}\right.
$$

Where $x, \mathrm{y}, z, w$ are the states and $u_{1}, u_{2}, u_{3}, u_{4}$ is the hybrid tracking controller.

If the four states $x, y, z, w$ of the system (6) can achieve tracking four-dimensional reference signals $r_{1}, r_{2}, r_{3}, r_{4}$, the states and reference signals will be synchronization respectively, and $\lim _{t \rightarrow \infty}\left\|e_{i}\right\|=0$ will be satisfied where $e_{i}(i=1,2,3,4)$ are tracking error states. The tracking error states are defined as

$$
\left\{\begin{array}{l}
e_{1}=x-r_{1} \\
e_{2}=y-r_{2} \\
e_{3}=z-r_{3} \\
e_{4}=w-r_{4}
\end{array}\right.
$$

The tracking error dynamics system can be obtained along formula (6) and formula (7). 


$$
\left\{\begin{array}{l}
\dot{e}_{1}=-2 x+y(z+3)+w+u_{1}-\dot{r}_{1} \\
\dot{e}_{2}=-2 \mathrm{y}+x(z-3)+u_{2}-\dot{r}_{2} \\
\dot{e}_{3}=z-x y+u_{3}-\dot{r}_{3} \\
\dot{e}_{4}=-m y+u_{4}-\dot{r}_{4}
\end{array}\right.
$$

We select the hybrid tracking controller which is defined by

$$
\left\{\begin{array}{l}
\mathrm{u}_{1}=x-y(z+3)-w+r_{1}+\dot{r}_{1} \\
u_{2}=\mathrm{y}-x(z-3)+r_{2}+\dot{r}_{2} \\
u_{3}=-2 z+x y+r_{3}+\dot{r}_{3} \\
u_{4}=2 y-w+r_{4}+\dot{r}_{4}
\end{array}\right.
$$

Along formula (8) and formula (9), the tracking error dynamics system can be simplified as:

$$
\dot{e}=-e
$$

We can know that the four characteristic values of the tracking error system are $^{[-1,-1,-1,-1]}$ from formula (10). Therefore, the tracking error dynamics system (8) will converge to the origin $(0,0,0,0)$ with an exponential contractions rate according to stability theory, and the four states $x, y, z, w$ of the system (6) can trace reference signals $r_{1}, r_{2}, r_{3}, r_{4}$ respectively with an exponential contractions rate

We select that the trace reference signal $r_{1}$ is a periodic signal $\cos t, r_{2}$ is a constant 3 , $r_{3}$ is a chaotic signal $\mathrm{X}_{1}$ of Chen chaotic system, and $r_{4}$ is a chaotic signal of Lü hyperchaotic system.

The Chen chaotic system [12] is given as below:

$$
\left\{\begin{array}{l}
\dot{x}_{1}=a\left(x_{2}-x_{1}\right) \\
\dot{x}_{2}=(c-a) x_{1}-x_{1} x_{3}+c x_{2} \\
\dot{x}_{3}=x_{1} x_{2}-b x_{3}
\end{array}\right.
$$

when $a=35, b=3, c=28$ and $x_{1}=1, x_{2}=1, x_{3}=1$ are selected, system (11) is chaotic. The projections of system (11) are illustrated in Figure 6.

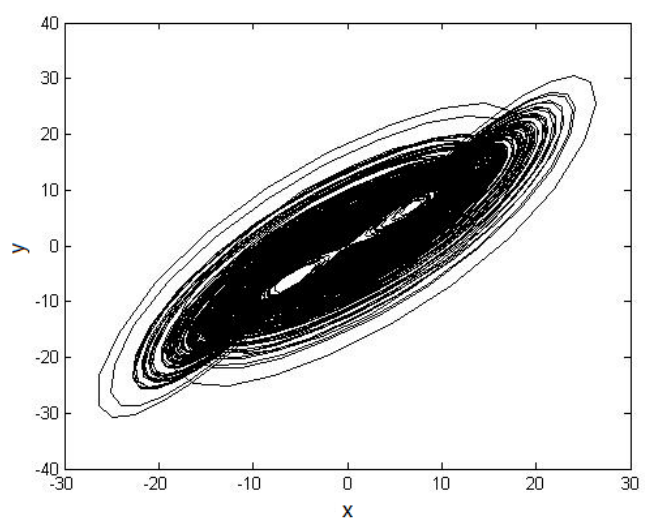

(a)

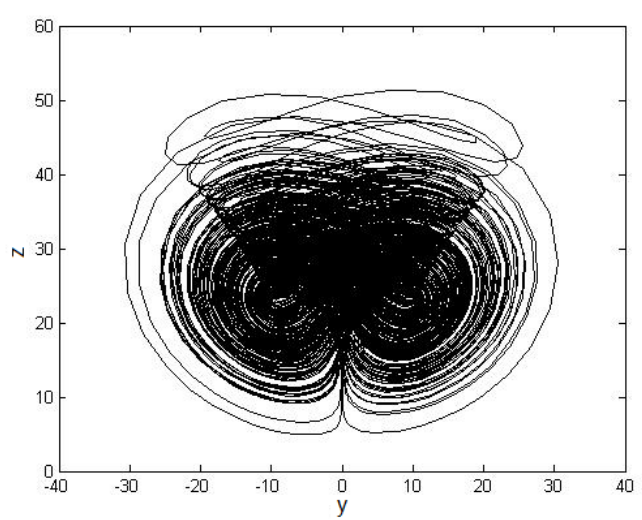

(b)

Figure 6. The Projection of the Chaotic Attractor of Chen Chaotic System (11) in (a) X-Y Plane, (b) Y-Z Plane

The Lü hyperchaotic system is given as below: 


$$
\left\{\begin{array}{l}
\dot{y}_{1}=10\left(y_{2}-y_{1}\right)+y_{4} \\
\dot{y}_{2}=5 y_{2}-4 y_{1} y_{3} \\
\dot{y}_{3}=-3 y_{3}+y_{1}^{2} \\
\dot{y}_{4}=0.5 y_{2}
\end{array}\right.
$$

when the initial condition $\mathrm{y}_{1}=-3, y_{2}=-4, \mathrm{y}_{3}=8$ and $\mathrm{y}_{4}=6$, system (12) is hyperchaotic. The projections of system (12) are illustrated in Figure 7.

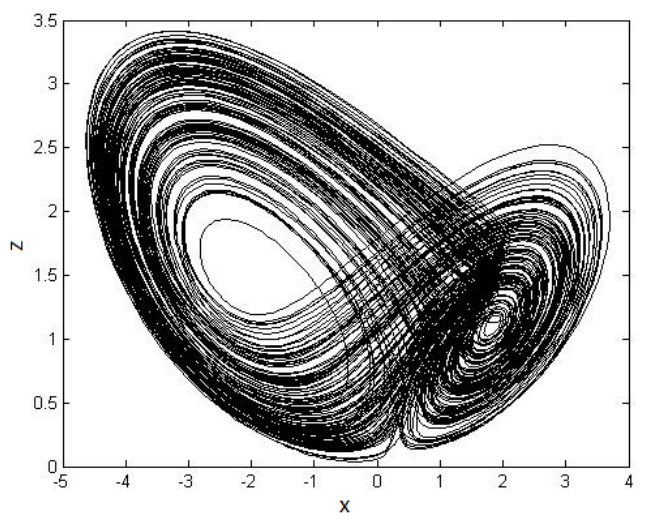

(a)

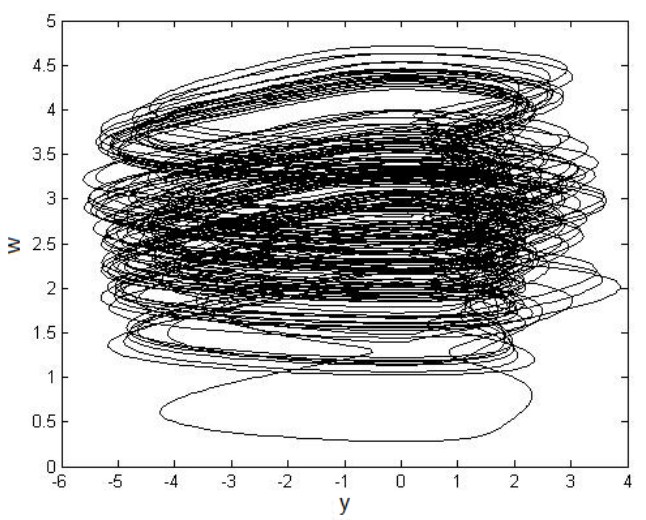

(b)

Figure 7. The Projection of the Hyper-Chaotic Attractor of Lü Hyperchaotic System (12) in (a) X-Z Plane, (b) Y-W Plane

We use MATLAB to make numerical simulation, use fourth-order Runge-Kutta method to solve error equation (8), step-size $h=0.001$ are selected, the simulation results are illustrated in Figure 8 and Figure 9. It can be seen that the time-history of tracking error will converge to zero in Figure 8. It shows that the states of fourdimensional coupling generator system (6) hybrid tracking four reference signals is achieved after $\mathrm{t} \leq 4$ seconds in Figure 8 . This means the validity and accuracy of the tracking using hybrid tracking control method.



(a)

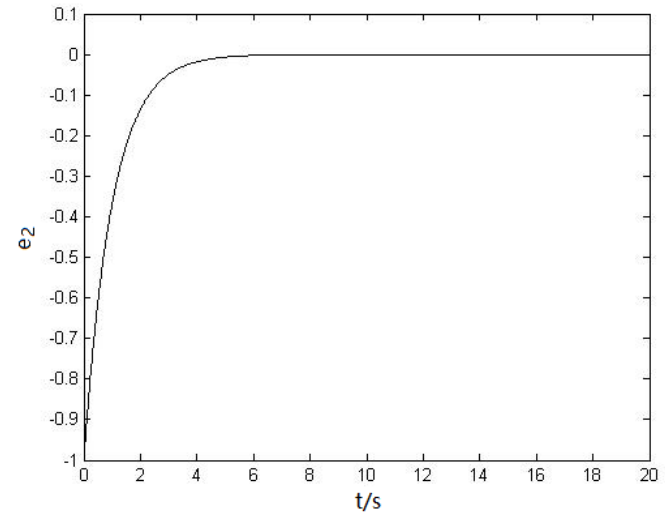

(b) 


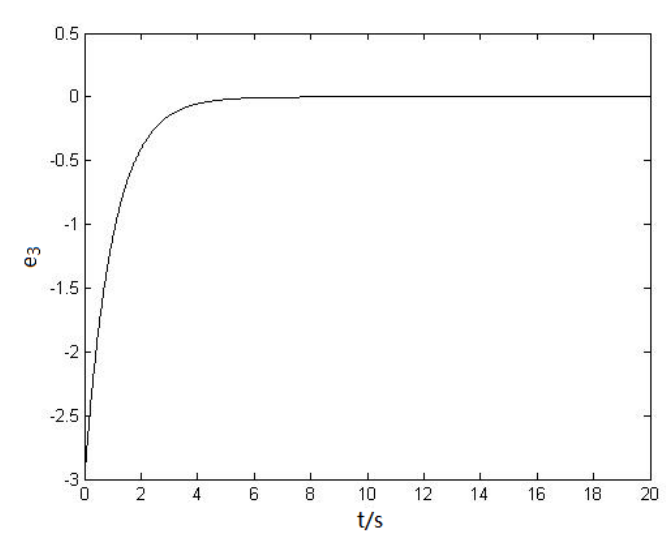

(c)

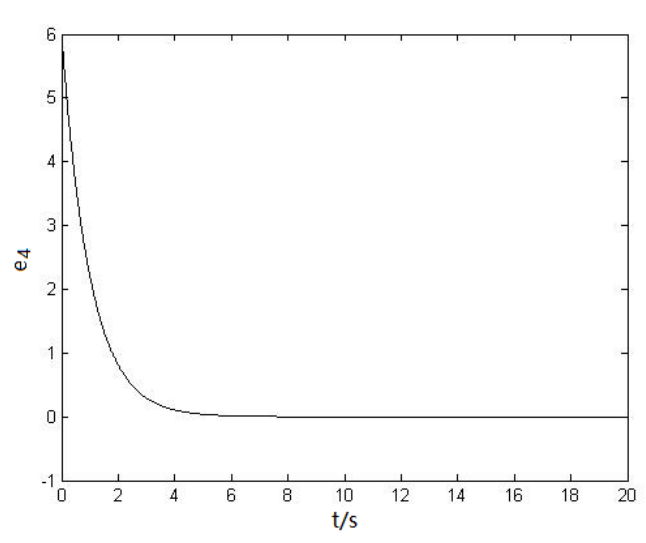

(d)

Figure 8. Synchronization Error Graph: (a) Time Evolution Graph of $e_{1}(t)$; (b) Time Evolution Graph of $e_{2}(t)$; (c) Time Evolution Graph of $e_{3}(t)$; (d) Time Evolution Graph of $e_{4}(t)$



(a)

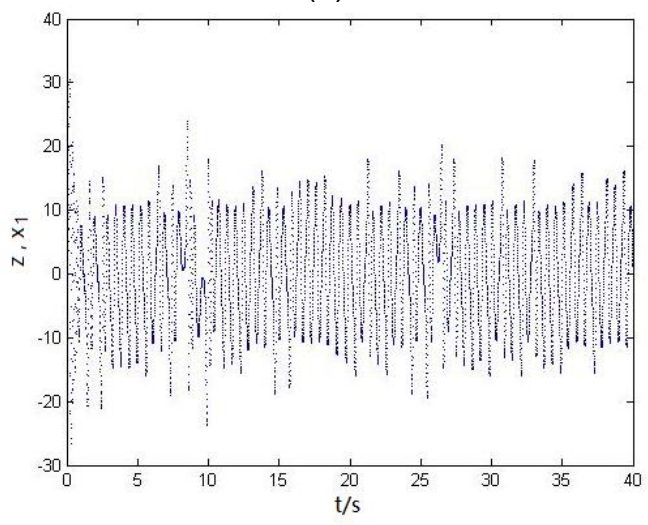

(c)

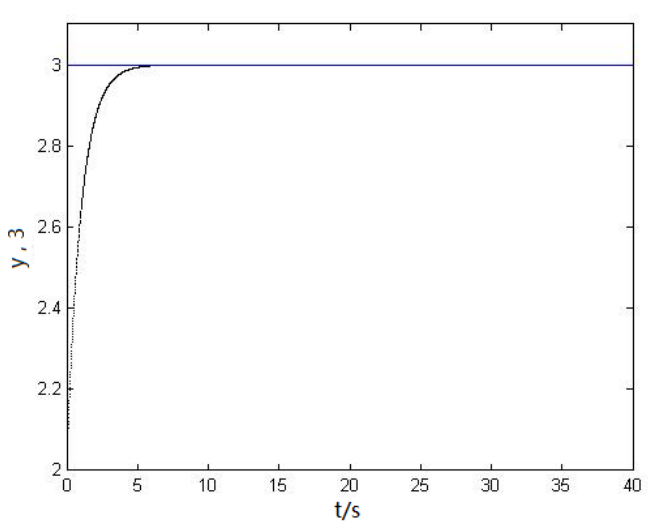

(b)

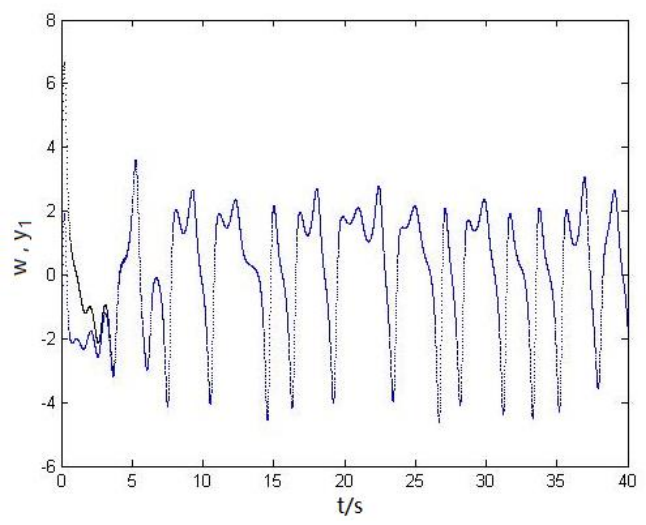

(d)

Figure 9. Time-Domain Waveform Between the Four States $x, \mathrm{y}, z, w$ of the System (6) and the Four Reference Signals: (a) $x$ Tracking $\cos \mathrm{t}$ (b) $\mathrm{y}$ Tracking Constant 3; (c) $z$ Tracking $x_{1}$; (d) $w$ Tracking $\mathrm{y}_{1}$ 


\section{Hyper-chaotic Synchronization}

When a new hyper-chaotic system is studied, its synchronization is often considered [13-37]. In this section, we use an adaptive synchronization method to make the driving system and response system achieving synchronization, and investigate synchronization for hyper-chaotic coupled dynamos system and hyper-chaotic Rossler system with different structures.

\subsection{Adaptive Synchronization}

We consider the driving system and response systems have the same structure, formula (1) is the driving system, the response systems can be described by

$$
\left\{\begin{array}{l}
\dot{x}_{1}=-2 x_{1}+y_{1}\left(z_{1}+3\right)+w_{1}+u_{1} \\
\dot{y}_{1}=-2 y_{1}+x_{1}\left(z_{1}-3\right)+u_{2} \\
\dot{z}_{1}=z_{1}-x_{1} y_{1}+u_{3} \\
\dot{w}_{1}=-2 y_{1}+u_{4}
\end{array}\right.
$$

In which $x_{1}, \mathrm{y}_{1}, z_{1}, w_{1}$ are the states and $u_{1}, u_{2}, u_{3}, u_{4}$ are adaptive controls. The synchronization error between driving system (1) and response systems (13) can be express as

$$
\left\{\begin{array}{l}
e_{1}=x_{1}-x \\
e_{2}=y_{1}-y \\
e_{3}=z_{1}-z \\
e_{4}=w_{1}-w
\end{array}\right.
$$

The synchronization error dynamics can be obtained along formula (1) and formula (13).

$$
\left\{\begin{array}{l}
\dot{e}_{1}=-2 e_{1}+y_{1} z_{1}-y z+3 e_{2}+e_{4}+u_{1}(t) \\
\dot{e}_{2}=-2 e_{2}+x_{1} z_{1}-x z-3 e_{1}+u_{2}(t) \\
\dot{e}_{3}=e_{3}-x_{1} y_{1}+x y+u_{3}(t) \\
\dot{e}_{4}=-2 e_{2}+u_{4}(t)
\end{array}\right.
$$

Therefore, we can know that the synchronization problems of four-dimensional hyperchaotic coupled dynamos system (1) and (13) can be transfer to the stability of synchronization error system (15) at the point $(0,0,0,0)$. The driving system can synchronize to the response system by selecting proper adaptive feedback control law $\mathbf{u}(t)=\left[u_{1}, u_{2}, u_{3}, u_{4}\right]^{T}$ to stabilize synchronization error system.

The adaptive feedback control law $\mathbf{u}(t)=\left[u_{1}, u_{2}, u_{3}, u_{4}\right]^{T}$ is defined by

$$
\left\{\begin{array}{l}
u_{1}(t)=-e_{3}\left(e_{2}+y\right)-e_{2} z-e_{4} \\
u_{2}(t)=-e_{3}\left(e_{1}+x\right)-e_{1} z \\
u_{3}(t)=e_{2}\left(e_{1}+x\right)+e_{1} y+k e_{3} \\
u_{4}(t)=q e_{4}
\end{array}\right.
$$

It is observing that the driving system (1) synchronize with the response system(13) with an exponential contractions rate when $k<-1, q<0$.

Because we select Lyapunov function which is considered as 


$$
V=\frac{1}{2}\left(e_{1}^{2}+e_{2}^{2}+e_{3}^{2}+e_{4}^{2}\right)
$$

By differentiate Lyapunov function $V$ along synchronization error system (15), we can get

$$
\dot{V}=e_{1} \dot{e}_{1}+e_{2} \dot{e}_{2}+e_{3} \dot{e}_{3}+e_{4} \dot{e}_{4}=-2 e_{1}^{2}-2 e_{2}^{2}+(k+1) e_{3}^{2}+q e_{4}^{2}
$$

Obviously, $\dot{V}$ is negative definite function when the parameters of controller $k<-1$, $q<0$ from (18). So according to Lyapunov stability theory, the synchronization error system (15) will converge to the origin $(0,0,0,0)$ with an exponential contractions rate, and the driving system (1) archive adaptive synchronize with the response system (13).

We use MATLAB to make numerical simulation, use fourth-order Runge-Kutta method to solve error equation (15), use controller $\mathbf{u}(t)$ to simulate the selfsynchronization of four-dimensional hyper-chaotic coupled dynamos system (1) and (13) which have the same structure, when $k=-2, q=-0.5$ and the initial condition $(x(0), y(0), z(0), w(0))=(-2,1,7,-2),\left(x_{1}(0), y_{1}(0), z_{1}(0), w_{1}(0)\right)=(3,2,8,3)$.

The numerical simulation results are illustrated in Figure 10. It shows that that the synchronization errors $e_{i}(t)$ will converge to zero and the driving system (1) and the response system (13) can achieve synchronization after $\mathrm{t} \leq 10$ seconds in Figure 10.

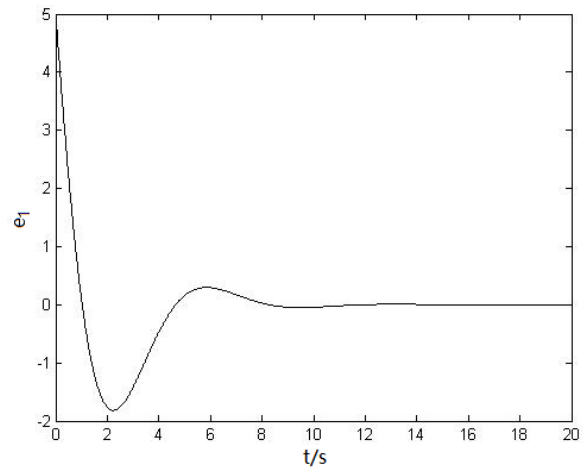

(a)

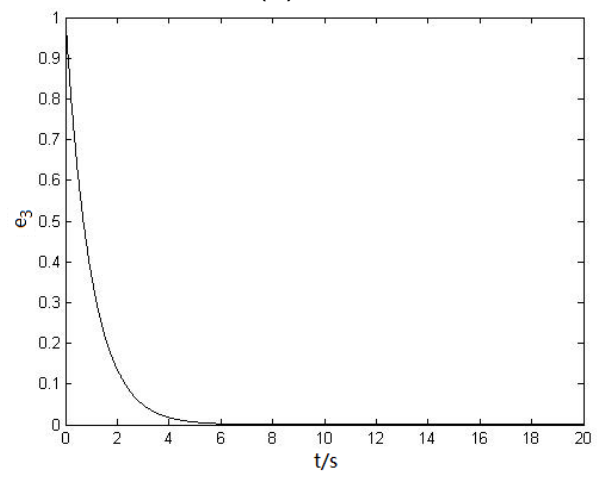

(c)

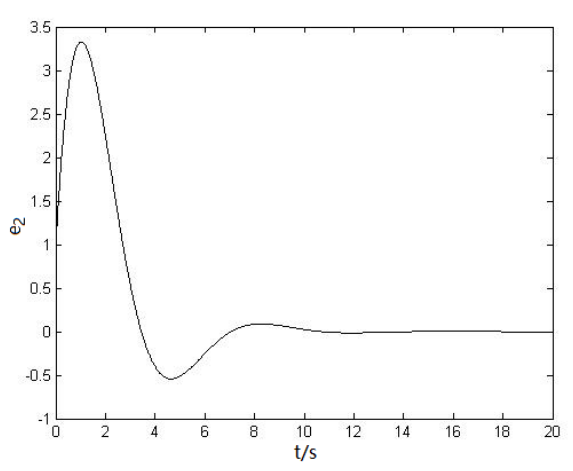

(b)

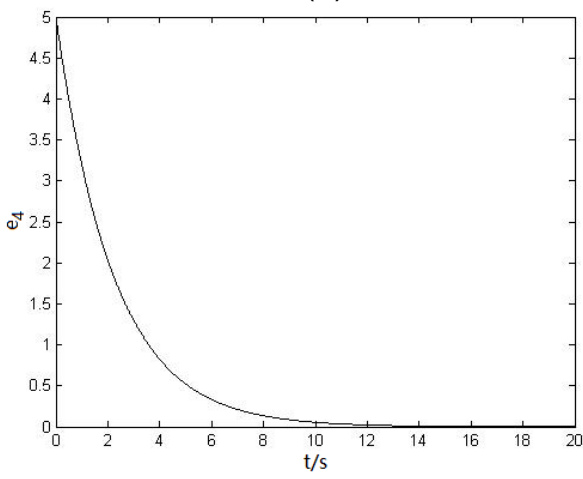

(d)

Figure 10. Synchronization Error Graph (a) Time Evolution Graph of $e_{1}(t)$;

(b) Time Evolution Graph of $e_{2}(t)$; (c) Time Evolution Graph of $e_{3}(t)$; (d) Time Evolution Graph of $e_{4}(t)$ 


\subsection{Synchronization for Hyper-Chaotic Systems with Different Structures}

When investigating synchronization for hyper-chaotic coupled dynamos system and hyper-chaotic Rossler system ${ }^{[38]}$ with different structures, we consider the driving system is formula (1) and the response systems is hyper-chaotic Rossler system which can be described by

$$
\left\{\begin{array}{l}
\dot{x}_{2}=-y_{2}-z_{2}+u_{1} \\
\dot{y}_{2}=x_{2}+0.25 y_{2}+w_{2}+u_{2} \\
\dot{z}_{2}=3+x_{2} z_{2}+u_{3} \\
\dot{w}_{2}=-0.5 z_{2}+0.05 w_{2}+u_{4}
\end{array}\right.
$$

In which $x_{2}, \mathrm{y}_{2}, z_{2}, w_{2}$ are the states and $u_{1}, u_{2}, u_{3}, u_{4}$ are control functions. Therefore, the synchronization error dynamics can be obtained by as

$$
\left\{\begin{array}{l}
\dot{e}_{1}=-e_{2}-e_{3}+2 x-4 y-z-w-y z+u_{1} \\
\dot{e}_{2}=e_{1}+0.25 e_{2}+e_{4}+4 x+2.25 y+w-x z+u_{2} \\
\dot{e}_{3}=3+e_{1} e_{3}+x e_{3}+z e_{1}-z+2 x z+u_{3} \\
\dot{e}_{4}=-0.5 e_{3}+0.05 e_{4}+2 y-0.5 z+0.05 w+u_{4}
\end{array}\right.
$$

We can know that the synchronization problems of four-dimensional hyper-chaotic coupled dynamos system (1) and hyper-chaotic Rossler system (19) with different structures can be transfer to the stability of synchronization error system (20) at the point $(0,0,0,0)$.

We select Lyapunov function which is defined by

$$
V=\frac{1}{2}\left(e_{1}^{2}+e_{2}^{2}+e_{3}^{2}+e_{4}^{2}\right)
$$

Theorem1. The hyper-chaotic coupled dynamos system (1) and hyper-chaotic Rossler system (19) can achieve synchronization and $\lim _{t \rightarrow \infty}\left\|e_{i}\right\|=0(i=1,2,3,4)$ when $\dot{V}_{\text {is negative }}$ definite function.

Proof.

We select the control functions which are defined by

$$
\left\{\begin{array}{l}
u_{1}=-e_{1}+e_{3}-2 x+4 y+z+w+y z \\
u_{2}=-1.25 e_{2}-e_{4}-4 x-2.25 y-w+x z \\
u_{3}=-e_{3}-3-e_{1} e_{3}-x e_{3}-z e_{1}+z-2 x z \\
u_{4}=0.5 e_{3}-1.05 e_{4}-2 y+0.5 z-0.05 w
\end{array}\right.
$$

By differentiate Lyapunov function $V$ along synchronization error system (20), we can get

$$
\dot{V}=e_{1} \dot{e}_{1}+e_{2} \dot{e}_{2}+e_{3} \dot{e}_{3}+e_{4} \dot{e}_{4}=-e_{1}^{2}-e_{2}^{2}-e_{3}^{2}-e_{4}^{2}
$$

Obviously, $\dot{V}$ is negative definite function from formula (22). So according to Lyapunov stability theory, the synchronization error system (20) will converge to the origin $(0,0,0,0)$ with an exponential contractions rate, and the hyper-chaotic coupled dynamos system (1) and hyper-chaotic Rossler system (19) achieve synchronization.

For the numerical simulation, the initial condition of hyper-chaotic coupled dynamos system (1) are selected as $(x(0), y(0), z(0), w(0))=(-2,1,7,-2)$, the initial condition of hyper-chaotic Rossler system (19) $\left(x_{2}(0), y_{2}(0), z_{2}(0), w_{2}(0)\right)=(-7,5,0,0)$ and step-size $h=0.01$ are selected, Figure 11 display the synchronization of system (1) and system 
(19)with different structures, which describes the time-history of the synchronization errors $e_{1}(t), e_{2}(t), e_{3}(t), e_{4}(t)$.



Figure 11. Time-History of the Synchronization Errors $e_{1}(t), e_{2}(t), e_{3}(t), e_{4}(t)$

\section{Conclusion}

Adaptive and hybrid tracking control methods is proposed based on four-dimensional hyper-chaotic coupling generator system model, design the suitable controller make the any variable of hyper chaos coupling generator system can track any reference signal; by choosing appropriate system control vector to make the system error stabilize to the origin within a few seconds, the drive and response system with the same structure achieve selfsynchronization at the same time; finally, this paper investigates synchronization for hyper-chaotic coupled dynamos system and hyper-chaotic Rossler system with different structures. According to Lyapunov stability theory and numerical simulation verification, the effectiveness of hyper chaos adaptive tracking control and synchronization methods is further illustrated. Adaptive tracking control and synchronization methods of this paper can equally applicable to other chaotic and hyper chaos system, and be further extended to multiple variables tracking and different structure synchronization.

\section{References}

[1] Ott E, Grebogi C, Yorke J A. Controlling chaos. Physical Review Letters. Vol. 64, No. 11, p. 11961199. (1990)

[2] Pecora L M, Carroll T L. Synchronization in chaotic system.Physical Review Letters.Vol. 64, No. 8, p. 821-824. (1990)

[3] Li LX, PENG HP, LU HB, GUAN XP. Control and synchronization of henon chaotic system. Acta Physical Sinica. Vol. 50, No. 04, p. 629-632. (2001)

[4] Wang XY, WU XJ. Adaptive control and synchronization of a coupled dynamos system. Acta Physical Sinica. Vol. 55, No. 10, p. 5077-5082. (2006)

[5] Peng JK, YU JN ZHANG L. Dynamics analysis and tracking control of a new complex lorenz-like chaotic system. Journal of Hebei Normal University (Natural Science Edition). Vol. 38, No. 06, p. 570577. (2014)

[6] Liu YJ. Combined synchronization control of hper-chaotic systems. College Mathematics. Vol. 27, No. 06, p. 19-22. (2011)

[7] Xie YY, Cai WL. A new four dimensional hyperchaotic coupled dynamos system and its hyperchaotic control. Journal of Chongqing University of Arts and Sciences. Vol. 35, No. 02, p. 19-22. (2016)

[8] Zhang XJ, Wu ZQ, Fang Zh. Approach to construction of hyperchaotic system. Computer Engineering and Applications. Vol. 50, No. 2, p. 92-98. (2014)

[9] Rossler O E. An equation for hyperchaos. Physics Letters A. Vol. 71, No. 2-3, p. 155-157. (1979)

[10] Wolf A, Swift J B, Swinney H L, Vastano J A. Determining Lyapunov exponents from a time series. Physics D:Nonlinear Phenomena. Vol. 16, No. 3, p. 285-317. (1985)

[11] Zhang HL, Min FH, Wang ER. The comparison for Lyapunov exponents calculation methods. Journal of Nanjing Nor Univ:Eng and Technol. Vol. 12, No. 1, p. 5-9. (2012) 
[12] Chen L, Wang D S. Adaptive tracking control of Chen system. Acta Physica Sinica, Vol. 56, No. 10, p. 5661-5664. (2007)

[13] Wei Y D, Zhou A J. Research on the anti-synchronization of a nolel five-dimensional hyperchaotic system. Ship Electronic Engineerring. Vol. 32, No. 11, p.33-36. (2012)

[14] Farmer J D, Ott E, Yorke J A. The dimension of chaotic attractors. Springer New York, (1982).

[15] Aguilar-Lopez R, Martinez-Guerra R, Perez-Pinacho CA. Nonlinear observer for synchronization of chaotic systems with application to secure data transmission. European Physical Journal-Special Topics. Vol. 223, No. 08, p. 1541-1548. (2014)

[16] Nijimeijer H, Mareels I. An observer looks at synchronization. IEEE Transaction on Circuits and Systems-I:Fundamental Theory and Applications. Vol. 44, No. 10, p. 882-890. (1997)

[17] Rosenblum M G. Pikovsky A S. Kurths J. From phase to lag synchronization in coupled chaotic oscillators. Physical Review Letters. Vol. 78 No. 22, p. 4193-4196. (1997)

[18] Akopov A, Astakhov V, Vadiasova T, Shabunin A, Kapitaniak T. Frequency synchronization in clusters in coupled extended systems. Physical Letters A. Vol. 334, p. 169-172. (2005)

[19] Karthikeyan R, Vaidyanathan S. Hybrid chaos synchronization of four-scroll systems via active control. J Electr Eng. Vol. 65 No. 2, p. 97-103. (2014)

[20] Vaidyanathan S, Volos C, Pham V T, Madhavan K, Idpwo B A. Adaptive backstepping control, synchronization and circuit simulation of a 3-D novel jerk chaotic system with two hyperbolic sinusoidal nonlinearities. Articles of Control Sciences. Vol. 24 No. 3, p. 257-285. (2014)

[21] Vaidyanathan S, Rasappan S. Adaptive single input control for synchronization of 4D Lorenz-Stenflo chaotic system. Arab J Sci Eng. Vol. 39, p. 2413-2426. (2014)

[22] Aghababa M P, Aghababa H P. A novel finite-time sliding mode control for synchronization of chaotic systems with input nonlineatity. Arabian Journal for Science and Engineering. Vol. 38 No.11, p. 32213232. (2013)

[23] Liu P, Liu S. Anti-synchronization between different chaotic complex systems. Physica Scripta. Vol. 83, No. 6, p. 264-297. (2011)

[24] Wu X J, Li S Z. Dynamics analysis and hybrid function projective synchronization of a new chaotic system. Nonlinear Dynamics. Vol. 69, No. 4, p. 1979-1994. (2012)

[25] Deng K B, Li J, Yu S. Dynamics analysis and synchronization of a new chaotic attractor. OptikInternational Journal for Light and Electron Optics. Vol. 125, No. 13, p. 3071-3075. (2014)

[26] Qiang J. Chaos control and synchronization of the Newton-Leipnik chaotic system. Chaos Solutions \& Fractal. Vol. 35, No. 4, p.814-824. (2008)

[27] Jia Z. Linear generalized synchronization of chaotic systems with uncertain parameters. Journal of Systems Engineering and Electronics. Vol. 19, No. 4, p.779-784. (2008)

[28] Jia Z, Lu J A, Deng G M, Generalized projective synchronization of a class of chaotic (hyperchaotic) systems with uncertain parameters. Chinese Physics. Vol. 16, No. 5, p.1246-1251. (2007)

[29] Gamal M M, Shaban A A, Ahmed A F. On chaos synchronization of a complex two coupled dynamos system. Chaos Solutions \& Fractal. Vol. 33, No. 1, p.178-187. (2007)

[30] Pham V T, Vaidyanathan S, Volos C, Jafari S, Kingni S T. A no-equilibrium hyperchaotic system with a cubic nonlinear term. Optik-International Journal for Light and Electron Optics.. Vol. 127, No. 6, p.3259-3265. (2016)

[31] Pham V T, Vaidyanathan S, Volos C, Jafari S, Kingni S T. A no-equilibrium hyperchaotic system with a cubic nonlinear term. Optik-International Journal for Light and Electron Optics. Vol. 127, No. 6, p.3259-3265. (2016)

[32] Volos C K, Kyprianidis I M, Stouboulos I N. Image encryption process based on chaoric synchronization phenomena. Signal processing. Vol. 95, No. 3, p.1328-1340. (2013)

[33] Wang S, Yang J, Luan H X. Controlling and synchronizing hyperchaotic Rossler system using backstepping design. Journal of Northeast Normal University(Natural Science Edition). Vol. 46, No. 2, p.69-73. (2013)

[34] Wang D X, Wu D Y. Research on the synchronizing of a novel five-dimensional hyper-chaotic system. Journal of Chongqing Normal University(Natural Science). Vol. 31, No. 6, p.87-91. (2014)

[35] Zhu H L. Optimal control and synchronization of a hyperchaotic system. Journal of Chongqing Normal University(Natural Science). Vol. 30, No. 3, p.65-68. (2013)

[36] Luo Y J, Yu Q, Zhang W D. Research on impulsive synchronization approach of parameter uncertain hyperchaotic systems with time-delay. Acta Physica Sinica. Vol. 60, No. 11, p.98-105. (2011)

[37] Qu S H, Wu S H, Yang Z H, The dynamic properties and adaptive synchronization of a new fourdimensional hyperchaotic system. Journal of Jiangxi Normal University:Natural Science Edition. Vol. 36, No. 1, p.87-91. (2012)

[38] Jiang N. Synchronization of hyperchaotic Lorenz system and hyperchaotic Rossler system in different structures. Journal of Shanxi adio \& TV University. Vol. 93, No. 04, p. 50-52. (2013) 


\section{Authors}



Yanyun Xie, theory research for nonlinear dynamics. Email: xieyanyun2008@126.com 
International Journal of Control and Automation Vol.10, No.4 (2017) 